\title{
Manufacturing of four-quadrant phase mask for nulling interferometry in the thermal infrared
}

Frederic Lemarquis, Michel Lequime, Gerard Albrand, Ludovic Escoubas, Jean-Jacques Simon, et al.

Frederic Lemarquis, Michel Lequime, Gerard Albrand, Ludovic Escoubas, Jean-Jacques Simon, Jacques Baudrand, Pierre Riaud, Daniel Rouan, Anthony Boccaletti, Pierre Baudoz, Dimitri Mawet, "Manufacturing of fourquadrant phase mask for nulling interferometry in the thermal infrared," Proc. SPIE 5250, Advances in Optical Thin Films, (25 February 2004); doi: 10.1117/12.513386 


\title{
Manufacturing of 4-Quadrant Phase Mask for nulling Interferometry in thermal infrared
}

\author{
Frédéric Lemarquis a , Michel Lequime a , Gérard Albrand ${ }^{\text {a }}$, Ludovic Escoubas ${ }^{\text {a }}$, Jean-Jacques Simon ${ }^{\text {a }}$, \\ Jacques Baudrand $^{\mathrm{b}}$, Pierre Riaud ${ }^{\mathrm{b}}$, Daniel Rouan $^{\mathrm{b}}$, Anthony Boccaletti ${ }^{\mathrm{b}}$, Perre Baudoz ${ }^{\mathrm{b}}$, Dimitri Mawet ${ }^{\mathrm{c}}$ \\ ${ }^{a}$ Institut Fresnel (UMR CNRS 6133), ENSPM, Dom. Univ. de S Jérôme, 13397 Marseille cedex 20, France \\ ${ }^{\mathrm{b}}$ LESIA, Observatoire de Paris-Meudon, 5 place J. Janssen, 92195 Meudon, France. \\ ${ }^{\mathrm{c}}$ IAGL, Lab. de Phy. Gén., Université de Liège, 17 Allée du 6 Août, B-4000 Sart-Tilman, Belgium
}

\begin{abstract}
The Four Quadrant Phase Mask is a key component for the design of advanced coronagraphs that may be used to search exo-planets. The validity of this concept has been demonstrated in the visible and need now to be demonstrated in the mid infrared. For this purpose, two components are manufactured for wavelengths 4.75 and $16.25 \mu \mathrm{m}$. This manufacturing requires the deposition of ZnSe layers using Ion Assisted Deposition, followed by a lift off process.
\end{abstract}

Key words: $\quad$ Phase mask, exo-planet detection, nulling interferometry, Ion Assisted Deposition, Lift-off

\section{INTRODUCTION}

\subsection{Scientific Context}

The understanding of planetary formation is one of the most exciting topic of this last decade. So far, the widely accepted scenario of planet formation was based on the observation of the Solar System itself (Lissauer et al. 1993), but in 1995, Mayor \& Queloz successfully detected the first "bona-fide" extra solar planet around the star 51 Peg. This planet turns out to be very "unusual" compared to the planets of the Solar System with a mass of roughly half of Jupiter but located 100 times closer to the star than Jupiter does. The presence of this peculiar planet has raised a lot of unanswered questions regarding its process of formation. To date, velocimetry technique has revealed more than a hundred of exoplanets. The velocimetry measures the reflex motion of the star and therefore only provide an indirect detection.

Among this consistent sample of exoplanets many of them are considered as "unusual" regarding their orbital parameters (large eccentricity, very close orbit) that could be explained with complex mechanisms such as planet migration. All this variety of planets definitely requires thorough investigations that can only be performed by direct imaging.

However, direct detection is still unachievable owing to the huge star/planet contrast $\left(\sim 10^{9}\right.$ in visible and near-IR $)$ and the small angular separation $(0.5$ " for a Jupiter-like planet at $10 \mathrm{pc})$.

The race towards high-contrast imaging has been motivated by the search of new worlds around distant stars and several clever concepts were recently investigated. Among these concepts, coronagraphy has undergone significant progress since the first coronagraphic instrument proposed by B. Lyot in the 30s. Indeed, such devices are required to attenuate the over shining stellar light. Achieving high-contrast also requires a very good optical quality and therefore space telescopes are usually preferred than ground-based telescopes to prevent the degradation by the atmospheric turbulence. The question of the optimal wavelength coverage is also critical and is a matter of trade off between the star/planet contrast and the background. In such a context, the James Webb Space Telescope could be precisely one of the most powerful observatory to search for extra solar worlds. 


\subsection{The James Webb Space Telescope and the MIRI instrument}

The James Webb Space Telescope (former NGST) will address many astrophysical topics from the understanding of the Universe structure to the birth and formation of primordial stars. It will be launched in august 2011 with a set of three instruments (NIRCAM, NIRSPEC and MIRI) to provide a wide spectral coverage from optical and near-IR $(0.6-5 \mu \mathrm{m})$ to mid-IR $(5-28 \mu \mathrm{m})$ wavelengths. The combination of a large aperture (about $6,5 \mathrm{~m})$ and unprecedented sensitivity will potentially make it a powerful facility for studying extra solar planets and brown dwarves. For that purpose, the mid-IR domain appears attractive since the star to planet contrast is significantly reduced and phase aberrations arising from the telescope itself are less critical at larger wavelengths.

A background analysis shows that the optimal spectral range for detecting and characterizing Jovian exoplanets is ranging from $9 \mu \mathrm{m}$ to $16 \mu \mathrm{m}$.

MIRI, the mid-IR instrument of JWST, is actually developed in a NASA-led partnership with a European consortium sponsored by the European Space Agency (ESA). The French part of this consortium, led by P.O. Lagage (CEA/Saclay), is deeply involved in the study of a high contrast coronagraph in what concerns numerical simulations and manufacturing of this device. The phase B of the MIRI study has started in May 2003. The Institut Fresnel and the Observatoire de Paris-Meudon are collaborating to fulfil a demonstration experiment of an advanced coronagraph in the mid-IR at cryogenic temperature: the Four-Quadrant Phase-Mask proposed by Rouan et al. (2000).

The principle of the Four Quadrant Phase Mask will be detailed in the next section, followed in section 3 by some experimental results in the visible range that demonstrate the high efficiency of this concept. The manufacturing of components for the mid infrared will be addressed in section 4 .

\section{PRINCIPLE OF THE FOUR QUADRANT PHASE MASK}

Back in 1939, B. Lyot first proposed to use an opaque mask in the focal plane of a telescope to improve the contrast of solar observations. This coronagraph was proven very efficient for systematic observations of the solar corona and was later turned into a stellar coronagraph to search for faint materials around bright stars such as circumstellar disks or lowmass companions. However, the stellar Lyot coronagraph is not efficient enough (in terms of angular separation and rejection factor) to allow the search for extra solar planets.

More recently, new concepts of high-contrast coronagraphs have been investigated and, in particular, Roddier \& Roddier (1997) have proposed to use a phase-mask instead of an amplitude mask. This device provides a $\pi$ phase-shift of half of the focal spot resulting in a self-destructive interference in the relayed pupil. As for the Lyot coronagraph, an appropriate stop is then inserted into the pupil plane to block the starlight which is spread out of the geometric pupil. The disk phase-mask is totally transparent and is also much smaller than a Lyot mask, allowing high-contrast imaging closer to the star.

Nevertheless, the disk phase-mask is highly chromatic regarding both the phase-shift and the mask size (0.5 $/ \mathrm{D})$. Rouan et al. (2000) solved the mask size wavelength dependence by turning the disk phase-mask into a Four-Quadrant PhaseMask (FQPM in the following). The principle of a coronagraph using such a phase mask is illustrated in figure 1. The concept and expected performance of the FQPM have been fully described in Rouan et al. (2000), Riaud et al. (2001). It has been demonstrated that for a circular pupil without any aberration the starlight attenuation is mathematically null (Rouan et al. 2001).

In more realistic conditions the performance of any coronagraph is always much worse than in the perfect case. Phasemask coronagraphs are indeed very sensitive to several factors: pupil shape, chromatism, wavefront distortions, residual jitter, manufacturing accuracy of the mask. For instance, Guyon et al. (1999) have reported an attenuation factor of about 16 using a disk phase-mask in a lab experiment. Regarding the FQPM, we achieved at the Observatoire de Meudon an rejection factor of 4500 (attenuation of the integrated energy) with a central peak attenuation of about 45000. This experiment is detailed in section 3. 


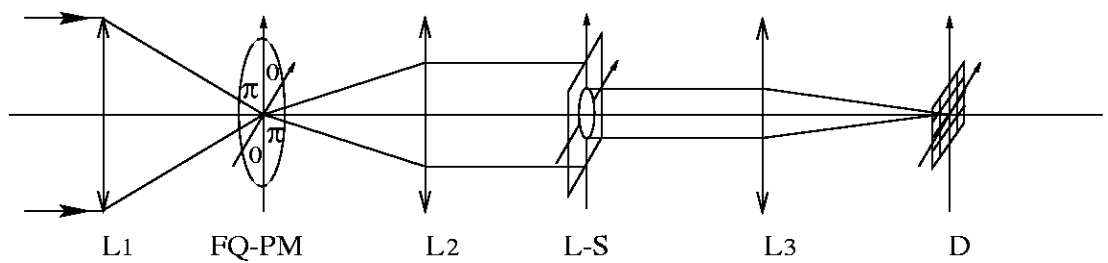

Figure 1: Concept of the Four-Quadrant Phase Mask. The FQPM is splitting the focal plane in 4 areas and shifts the phase of 2 quadrants by $\pi$. This simply changes the sign of the complex amplitude in the corresponding quadrants and results in a destructive interference in the re-imaged pupil.

An appropriate stop (L-S) selecting the dark pupil remove all of the diffracted starlight.

\section{LABORATORY RESULTS}

\subsection{First tests in the "Visible"}

After a period dedicated to numerical simulations assessing the FQPM behaviour (Riaud, 2001) an experimental photometric test was then undertaken aiming to put under trial a first FQPM prototype.

Figure 2 shows an illustration of a FQPM. The $\pi$ phase shift is obtain at any wavelength that verifies equation $(n-1) \cdot d=k . \lambda / 2$, where $n$ is the refractive index of the material, $d$ the thickness of the quadrants and $k$ an odd integer. A first component was manufactured by SAGEM/REOSC: a C2035 glass substrate coated with an $\mathrm{Al}_{2} \mathrm{O}_{3}$ four quadrant geometry by way of a classical lift-off deposition technique (see fig.3).

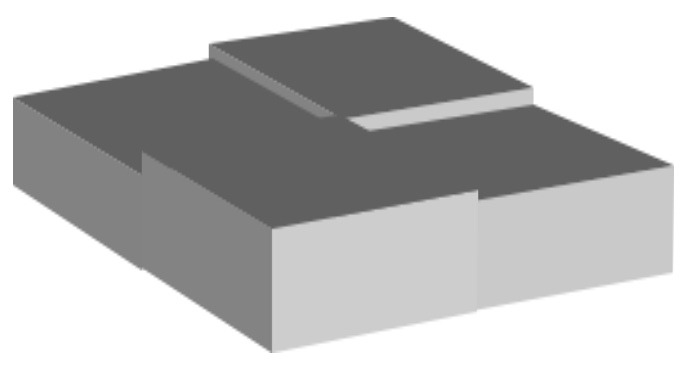

Figure 2: Representation of a FQPM. The $\pi$ phase shift is obtained for wavelengths that verifies $(n-1) d=k \lambda / 2$ where $\mathrm{k}$ is an odd integer

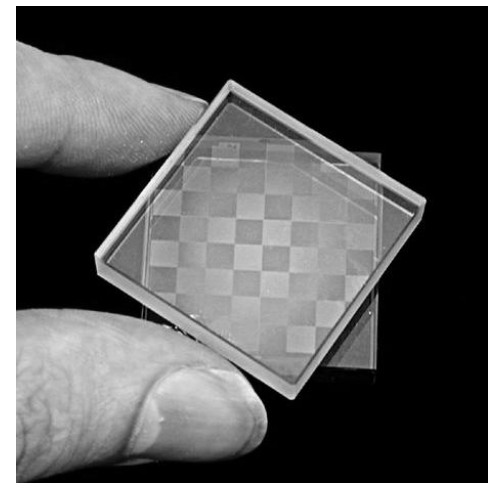

Figure 3: FQPM prototype manufactured by SAGEM/REOSC operating at $640 \mathrm{~nm}$. An array of $(7 \times 7)$ masks were manufactured on a single substrate.

The laboratory set-up for the tests reproduces the basic optical arrangement presented in the previous section. The selected monochromatic $(\Delta \lambda=2 \mathrm{~nm})$ light source, a $635 \mathrm{~nm}$ nominal wavelength laser diode, can be conveniently tuned on a small spectral range to match with precision the effective optical path difference corresponding to the $533 \mathrm{~nm}$ thick $\mathrm{Al}_{2} \mathrm{O}_{3}$ deposited layer. Light is injected onto the optical assembly first lens by way of a $4 \mu \mathrm{m}$ core diametermonomode fibre. Owing to the high surface quality of its optical components the wavefront distortions of this assembly are minimized and the Strehl ratio of the image delivered on the mask was measured to be $>99.5 \%$. When the fibre image is perfectly centred onto the FQPM the light is rejected outside the system pupil aperture where it is filtered by a reduced $(75 \%)$ aperture stop. The resulting faint coronagraphic image produced by the light residuals passing through the stop is compared with the incident parent image. The peak to peak and total energy attenuations were respectively 
measured to be 45000 and 4500. After an additional pos t-processing consisting in a centro-symmetrical subtraction of the quadrant the speckle level in the coronagraphic image was still reduced to a speckle level of about $10^{-6}$ of the parent image peak intensity (see fig. 4). All these results are presented and discussed in the details in Riaud et al. (2003).

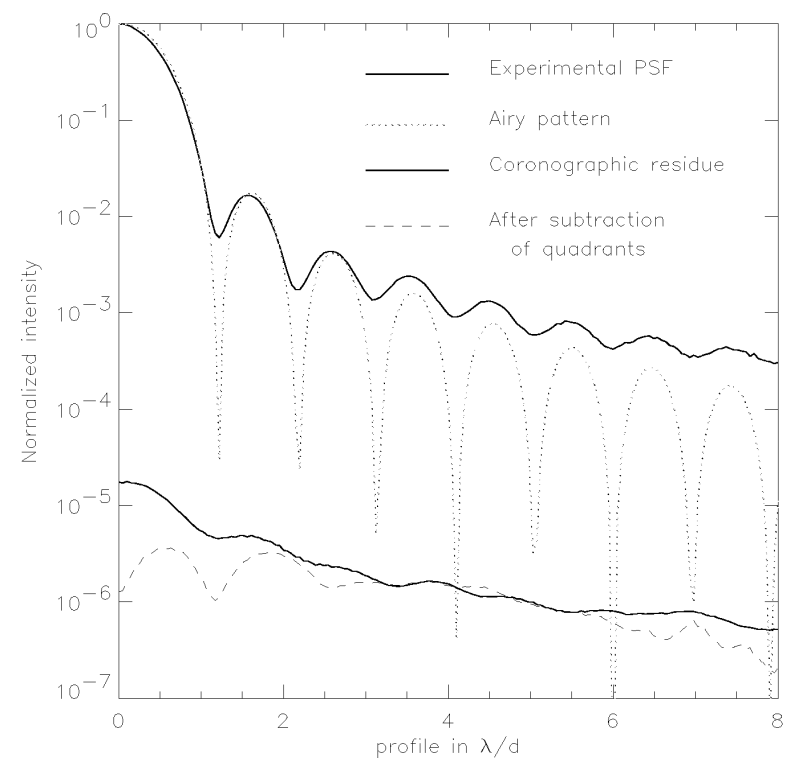

Figure 4: Normalized radial profiles obtained for the experimental PSF (solid line), compared to the theoretical Airy pattern (dotted line). The two curves below present the coronagraphic residuals before (solid line) and after (dotted line) centro-symmetrical subtraction. All curves are azymuthally averaged.

\subsection{Detection capability}

The efficiency of the FQPM coronagraph was also demonstrated by a simple experiment simulating the direct detection of a faint companion in the vicinity of an intense star. The faint companion was "fabricated" by way of a double reflection through a AR coated high quality surface silica substrate. The intensity ratio between the incident image and its ghost was about 15000 corresponding to an astronomical magnitude difference of 10.5 . Figure 5 presents the resulting images of this arrangement before and after passage through the FQPM. In the first case the "companion" is hidden behind the second bright ring of the "star" image. In the second case it is easily detected at $20 \sigma$ above the "star residual".

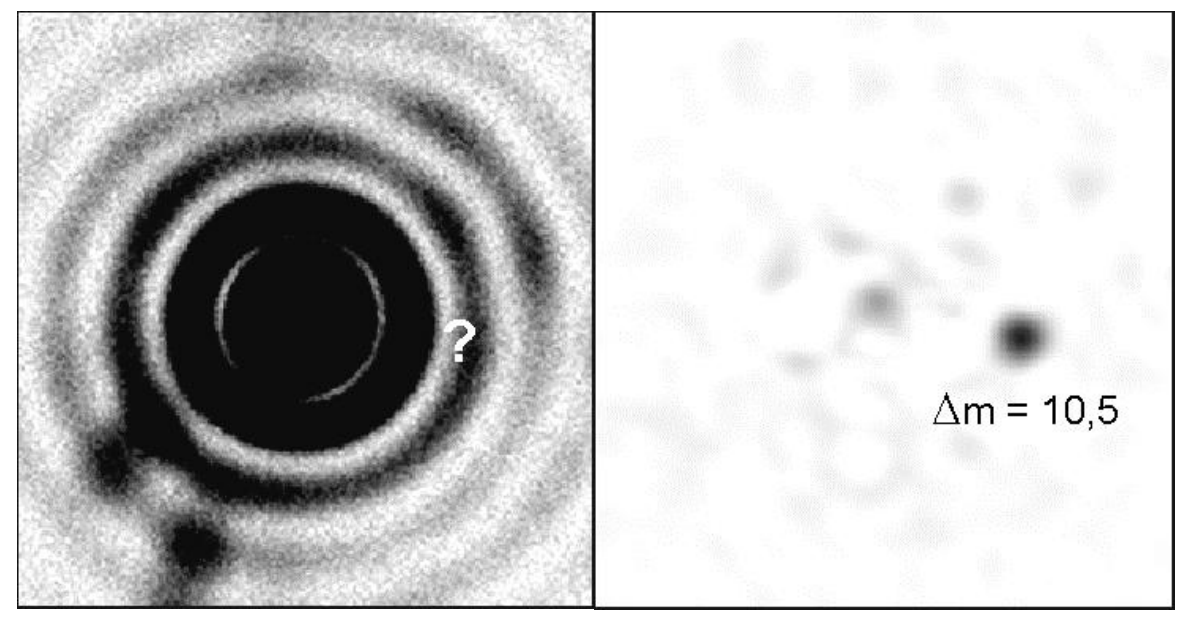

Figure 5: Experimental result of the direct detection of a faint "companion" in the vicinity of a bright "star". The astronomical magnitude difference between the two incident images is equal to 10.5 corresponding to a flux ratio of 15000 . 


\subsection{Operating wavelength}

It is often necessary to determine the effective optical path difference of the FQPM quadrant step with a higher precision than the one provided by the manufacturing process alone. For that purpose a low resolution spectroscopic device was implemented on the test bench optical arrangement in the parallel beam before the stop and the monochromatic source was replaced by a $3400 \mathrm{~K}$ white halogen lamp. The coronagraphic image is now dispersed and the recorded resulting continuous spectrum exhibits a minimum corresponding to the maximum rejection ratio of the tested mask, thus defining its specific operating wavelength. Wavelength calibration is performed on several reference lines (He-Ne and Nd-Yag laser lines) with a nanometric precision. This facility proved to be particularly useful with the SAGEM mask which experienced troublesome shifts in wavelength. Indeed this behaviour was later explained by the porous nature of the $\mathrm{Al} 2 \mathrm{O} 3$ deposited layer and the consequent variations of its refractive index with environmental conditions and mask cleanness.

\section{INFRARED DEVELOPMENT FOR THE JWST / MIRI}

\subsection{Strategy and manufacture}

In the context of the JWST/MIRI project we have proposed to implement the FQPM in the imager module as an effort to improve the coronagraphic instrument expected performance which relied solely in its original baseline on the simple sharp edge Lyot mask. The excellent first laboratory results obtained with a 'visible' mask were certainly a first step to win the ticket on-board but we were also asked to undertake a feasibility study dedicated to the mid-IR band. We are currently involved in this demonstration phase where it is planned to fabricate two FQPM centred respectively at 4.75 and $16.25 \mu \mathrm{m}$ and to assess their mechanical resistance and their photometric performance in terms of light rejection capability. The Institut Fresnel has been selected as the sub-contractor for the manufacturing of these components.

Several transparent materials can be used in this spectral range, such as ZnSe, Ge or CdTe. We selected ZnSe, mainly because of its transparency from the visible to the IR up to $17 \mu \mathrm{m}$. This has the advantage to cover the full bandwidth requirement for MIRI and also to allow the precise measurement of the components operating wavelength using the visible test bench developed at the Observatoire de Meudon (see section 4.2).

In order to form the 4 quadrants on the substrate as illustrated in figure 1, two solutions are possible, which are either to etch two quadrants in the substrate, or to deposit a $\mathrm{ZnSe}$ layer on two quadrants. Since the etching of $\mathrm{ZnSe}$ is not frequent, with very few results in the literature, we preferred the deposition solution.

Obviously, the thickness of the layer ( $d)$ depends on both the working wavelength of the component $(\lambda)$ and the substrate refractive index at this wavelength $(\mathrm{n})$, according to the equation $(\mathrm{n}-1) \cdot \mathrm{d}=\lambda / 2$.

For components working at 4.75 and $16.25 \mu \mathrm{m}$, the required thicknesses are about 1.8 and $6.25 \mu \mathrm{m}$.

In addition to this thickness constraint, the quadrants must be deposited with the required geometrical accuracy. More precisely, the transition zone between two adjacent quadrants must be less than $10 \mu \mathrm{m}$. To obtain this result, we used a classical lift off technique, as illustrated in figure 6. Because of the photoresist layer, the substrate temperature should not be too high during the whole process and especially during the deposition of $\mathrm{ZnSe}$. However, we know that $\mathrm{ZnSe}$ layers deposited at ambient temperature with a classical evaporation process have poor mechanical and climatic behaviour because of a low packing density, which is not compliant with space environment. To overcome this difficulty, we used an Ion Assisted Deposition process (IAD). Indeed, this high energy deposition technique is known to give dense layers while the substrate temperature does not exceed $70^{\circ} \mathrm{C}$.

At last, in order to improve the transmittance of the component and to suppress parasitic light beams, the components must be antireflection coated on both sides. A two layer AR design gives satisfactorily results ( $\mathrm{R}<0.5 \%$ ) considering that the component must work at in a short bandpass $(\lambda / \Delta \lambda=10)$. In addition to $\mathrm{ZnSe}$, we used $\mathrm{YF}_{3}$ as a low index material for these coatings. 


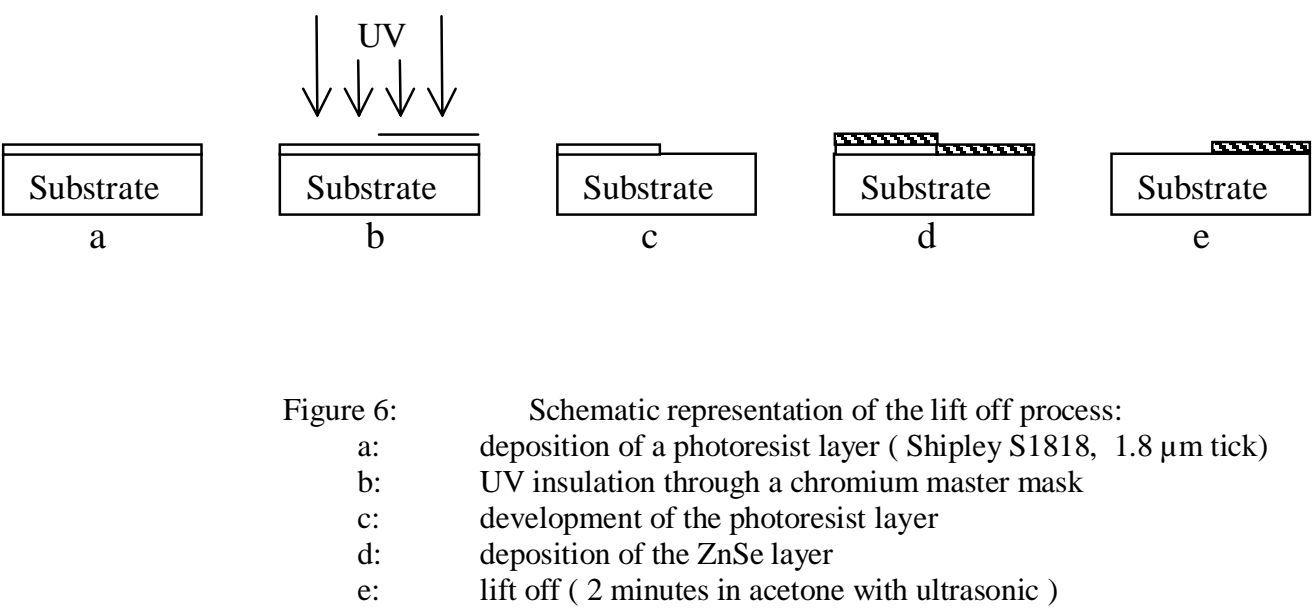

We first determined adequate deposition parameters for both $\mathrm{ZnSe}$ and $\mathrm{YF}_{3}$. Indeed, the Ion Assisted Deposition technique is not frequently used for such materials, but mainly recommended for oxide materials. For both materials, we used only argon for the assistance ion beam. Several trials were performed on glass and silica substrates, varying the ion gun parameters, to minimise the absorption level.

Concerning $\mathrm{ZnSe}$, we essentially met difficulties with layer adhesion on silica substrates, especially for large thicknesses. Fortunately, we did not notice this problem on ZnSe substrates, as required for the FQPM manufacturing, even for layer thicknesses up to $6 \mu \mathrm{m}$ for the component centred at wavelength $16.25 \mu \mathrm{m}$. The packing density of IAD ZnSe layers was estimated to 0.98 by optical measurements performed in ambient medium and in vacuum at the Observatoire de Meudon.

Concerning $\mathrm{YF}_{3}$, we observed, as mentioned in the literature for fluoride materials, that the layer absorption increases with the ion beam power. As a consequence, a low assistance level was used for the deposition of this material.

In a second step, we optimized the lift off process for ZnSe. Notice that the thicknesses of the photoresist and $\mathrm{ZnSe}$ layers are similar for the $4.75 \mu \mathrm{m}$ FQPM.

Figures 7, 8 and 9 give an illustration of the results at the junction of the four quadrants for a component working at $4.75 \mu \mathrm{m}$.

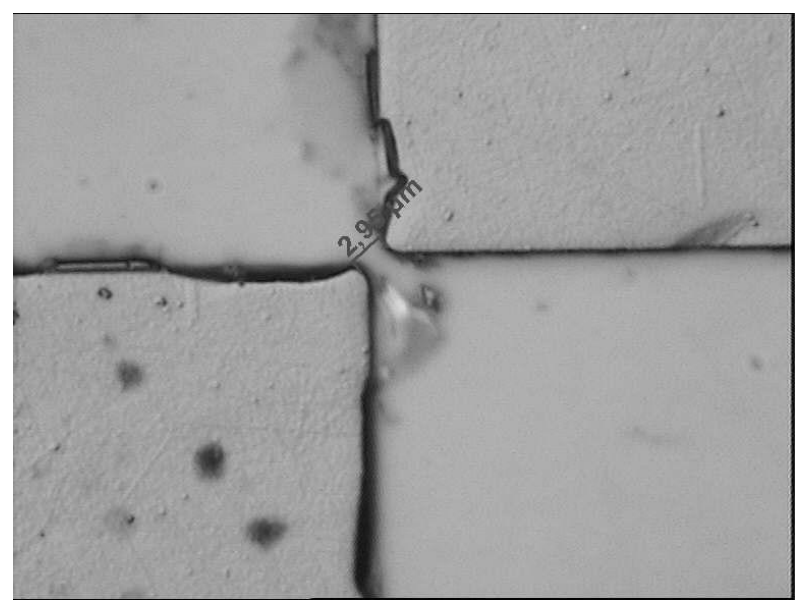

Figure 7: Nomarsky microscopy of a $4.75 \mu \mathrm{m}$ phase mask. The distance between opposite quadrants is about $3 \mu \mathrm{m}$ 


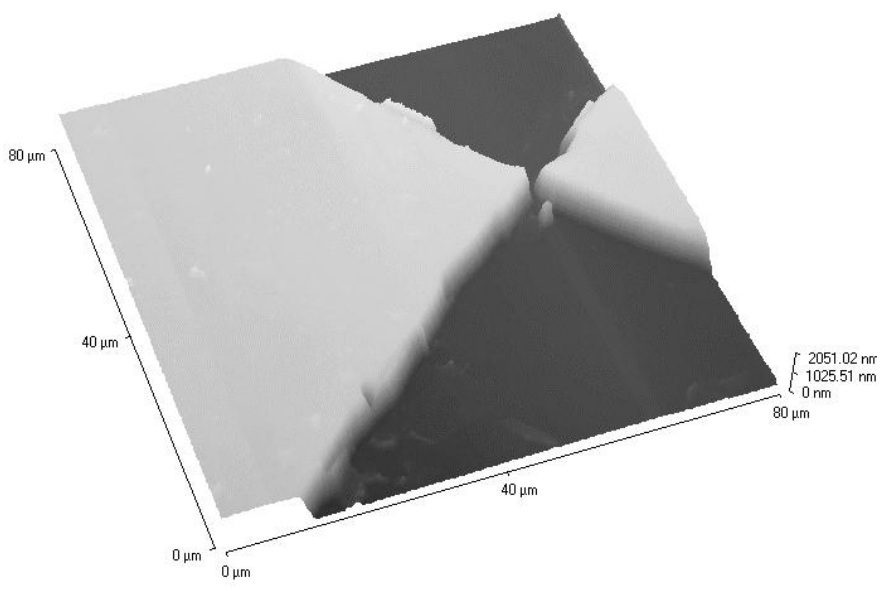

Figure 8: Atomic Force Microscopy of the FQPM. The size of the image is $80 * 80 \mu \mathrm{m}$

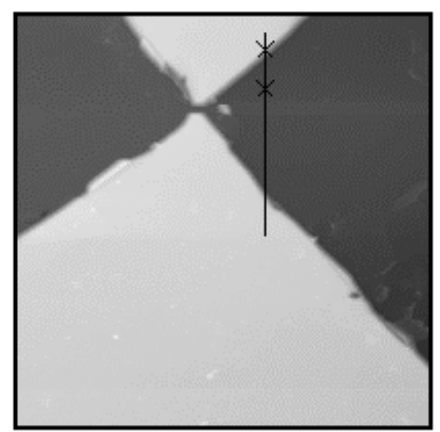

\begin{tabular}{lccc} 
& & Line1 \\
& $X(\mu \mathrm{m}]$ & $Y(\mu \mathrm{m})$ & $Z(\mathbf{n m}]$ \\
Point1: & 3.2 & & 1702.1 \\
Point2: & 10.9 & & 228.1 \\
Diff: & 7.6 & & -1473.9 \\
Length: & \multicolumn{3}{c}{$7.776 \mu \mathrm{mm}$} \\
Pt Angle: & \multicolumn{3}{c}{$10.93^{\circ}$} \\
\end{tabular}

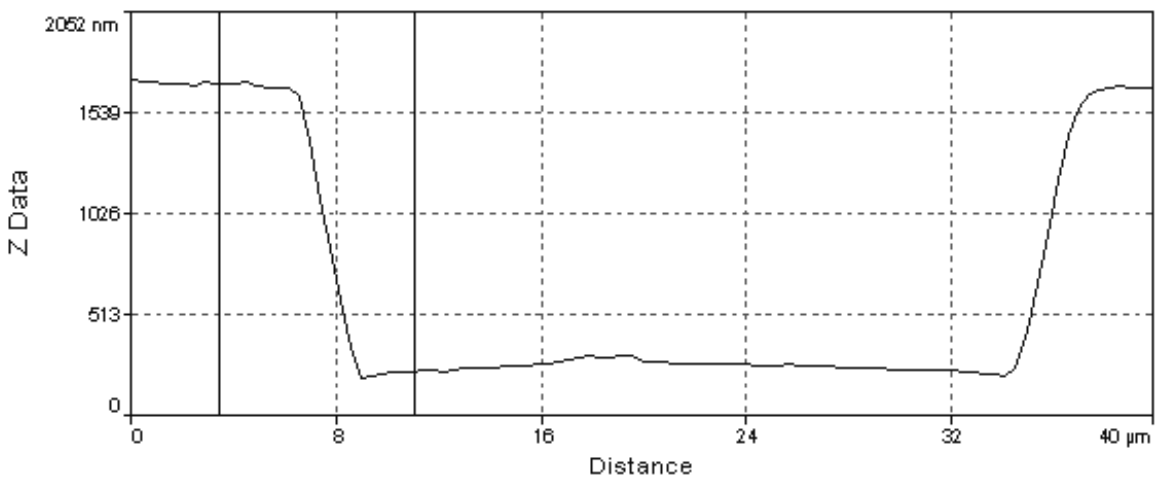

Following these results, two FQPM were manufactured and antireflection coated for wavelength 4.75 and $16.5 \mu \mathrm{m}$. For both masks the deposition is made on ZnSe square plates, $15 \mathrm{~mm}$ aside, $4 \mathrm{~mm}$ thick, polished with a high surface quality $(\lambda / 30 \mathrm{rms}$ at $633 \mathrm{~nm})$. Fig 10 shows a picture one of these FQPM which are presently under control in the laboratory. 


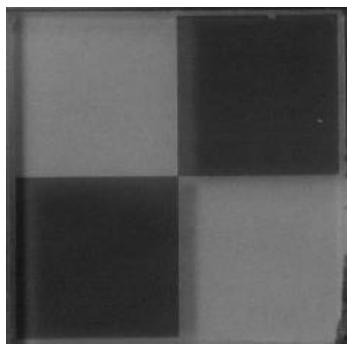

Figure 10: Picture of the $16.2 \mu \mathrm{m}$ FQPM

manufactured by the Institut Fresnel.

The 4 quadrant deposited layer and the

substrate are $\mathrm{ZnSe}$. The size of the mask is

$15 \mathrm{~mm}$ aside

\subsection{Laboratory controls}

A visual inspection of the masks under high magnification is first performed allowing to control their general cosmetic aspect as well as the width and quality of the transition between quadrants. The next important step is then dedicated to the precise measurement of the operating wavelength for each mask. This delicate operation is done on the 'visible' test assembly completed with its spectroscopic capability. Indeed, according to the general phase shift relation $\mathrm{k} . \phi=2 . \pi / \lambda . d .\left(\mathrm{n}_{(\lambda)}-1\right)$, with $\mathrm{k}$ an odd integer, we take advantage of the fact that a mask designed to operate in the mid-IR at the first order can also work at lower wavelengths defined by this relation and for the next upper orders. This is what is presented in the figure 11 where the spectral profiles for the two masks exhibit deep minima corresponding to a $\pi$ phase shift for successive orders. The related measured wavelengths for each minimum provide then series of equations that ultimately allow to calculate the first order operating wavelength. For both masks the specification $\left(\lambda_{\mathrm{o}} \pm\right.$ $3 \%$ ) was found to be respected with the first FQPM centred at $4.881 \mathrm{~nm}$ and the second at $16.314 \mathrm{~nm}$ (at $300 \mathrm{~K}$ ).
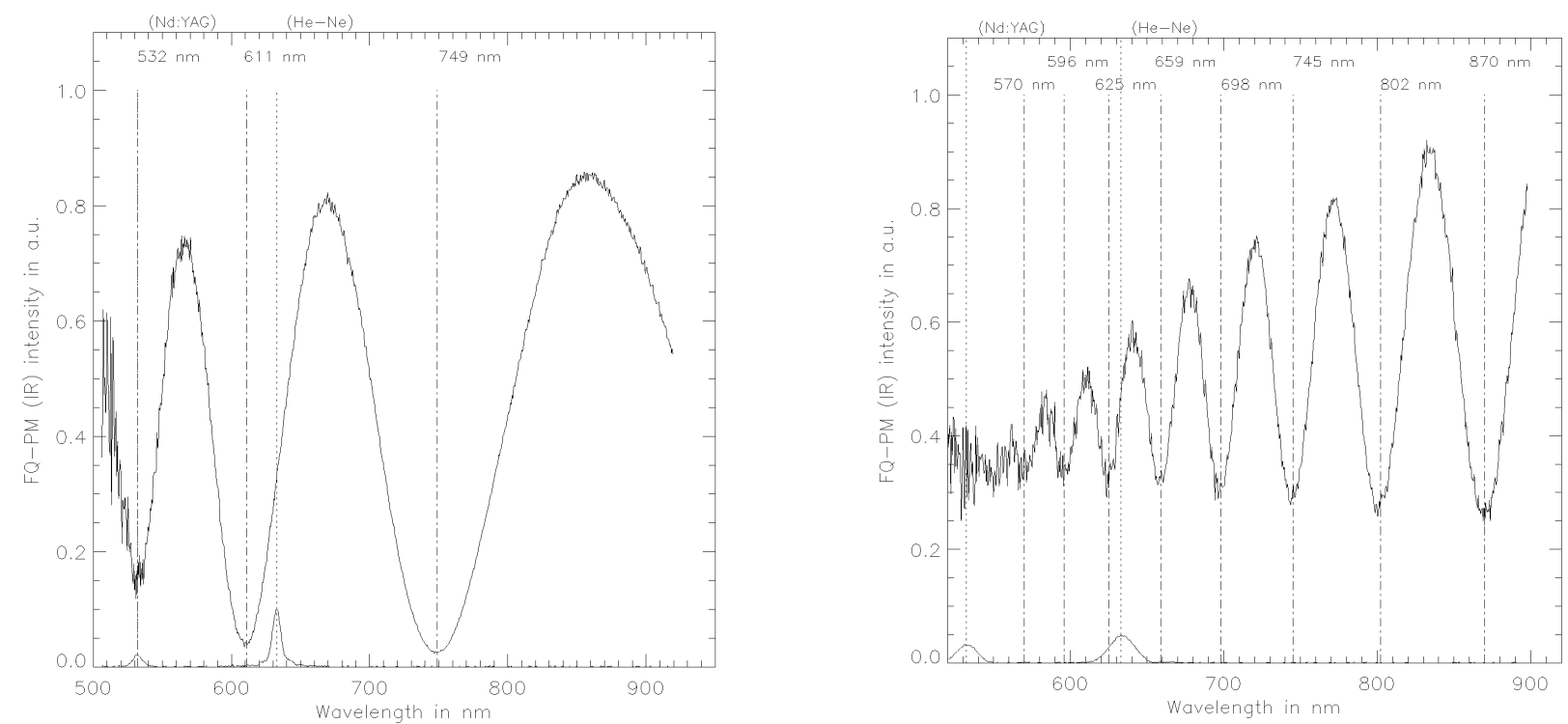

Figure 11: Photometric profiles of low resolution spectroscopy of two IR masks manufactured by the Institut Fresnel. Several well detected orders in the 'visible' allow to derive accurately their respective operating wavelength at the first order in the IR. Here $4.881 \mu \mathrm{m}$ (left) and 16,314 $\mu \mathrm{m}$ (right) with a precision of $\pm 3 \mathrm{~nm}$.

After determination of the operation wavelength the masks are put under severe conditions close to space flight qualification tests and we check the integrity of the deposited layers, quadrants and AR coatings.

At last the final action consists in measuring the effective rejection factor of the FQPM placed in a coronagraph at low temperature. For this a dedicated $35 \mathrm{~K}$ cryogenic assembly as been implemented including a basic coronagraphic optical arrangement making use of $\mathrm{ZnSe}$ lenses and $\mathrm{CaF} 2$ windows (fig.7). For MIRI the requirement for the total rejection factor (given with respect of the image total energy) is only of 500, a limitation mainly due to the JWST delivered 
wavefront errors, pupil features and guiding jitter. This is this threshold that must at least be reached during our laboratory tests. This will be done with the $4.75 \mu \mathrm{m}$ mask through a spectral bandpass of about $0.5 \mu \mathrm{m}$ (because of the intrinsic limitation of our detector array the $16.25 \mu \mathrm{m}$ mask will not be tested).

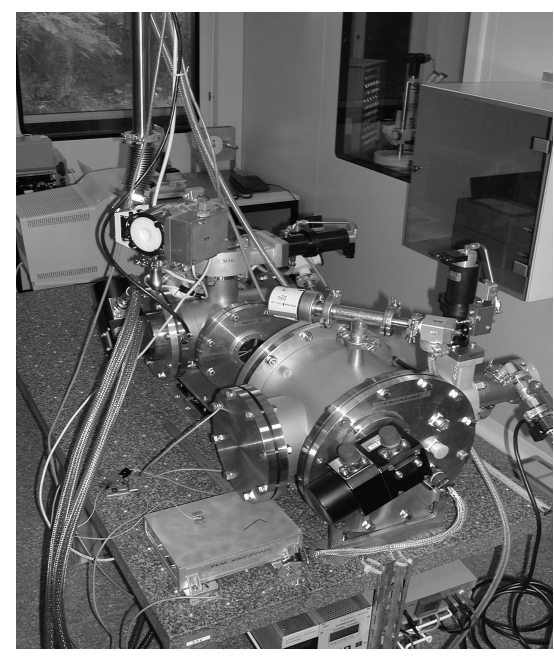

\author{
Figure 7: Cryogenic \\ facility for testing the \\ attenuation performance \\ of the $4.75 \mu \mathrm{m}$ FQPM
}

\title{
5. CONCLUSION AND FUTURE WORK
}

Following previous results that demonstrated the efficiency of the FQPM concept in the visible range, two FQPM designed for mid infrared wavelengths 4.75 and $16.25 \mu \mathrm{m}$ were manufactured at Institut Fresnel. In order to obtain sharp transitions between quadrants, lift off techniques are mandatory, which requires a low temperature deposition process. In order to fulfil this requirement as well as the necessity to obtain mechanical properties that are compliant with space environment, these components were manufactured using Ion Assisted Deposition. The quadrants geometry, as well as their thickness are in agreement with initial specifications.

At present days, the $4.75 \mu \mathrm{m}$ FQPM is under evaluation at the Observatoire de Meudon. In particular, the attenuation performance will be measured at $35 \mathrm{~K}$.

The next step for such phase mask coronagraph is the achromatisation of the $\pi$ phase-shift. Indeed, for high contrast imaging up to $10^{5}$, dedicated for instance to exo-planetary detection, achromatisation on a large bandwidth is mandatory. Today various technological schemes have been assessed: dispersive plates, half-wave plates and multicoating solutions.

In that context a study has been initiated for the European ground-based VLT (ESO) where our team is contributing to address the feasibility of a Planet Finder instrument. We are specifically investigating the use of half-wave plates to provide an achromatisation in the near-IR from $0.7 \sim 2.5 \mu \mathrm{m}$ with a single FQPM. This solution provides high phase residuals around $\pi$ up to $0.1 \mathrm{rad} \mathrm{rms}$ which is consequent with the foreseen high order adaptive optics correction on the VLT.

The use of such coronagraph on a space interferometer to search for terrestrial planets is also envisaged at longer term. In that case the residuals level on large spectral band must be less than $4.10^{-3} \mathrm{rad} \mathrm{rms}$, a requirement that could theoretically be satisfied with the multi-coating reflection FQPM (see same session: Lemarquis. F. \& Riaud. P.).

\section{REFERENCES}

Dubreuil D. et al., 2002, Proc. SPIE 4850, 564

Guyon O. et al., 1999, PASP 111, 1321

Lissauer J.J.,1993, ARA\&A vol 31, 129

Riaud P. et al., 2001, PASP 113, 1145

Riaud P. et al., 2003, PASP 115, 712

Rouand D. et al., 2000, PASP 112, 1479 